
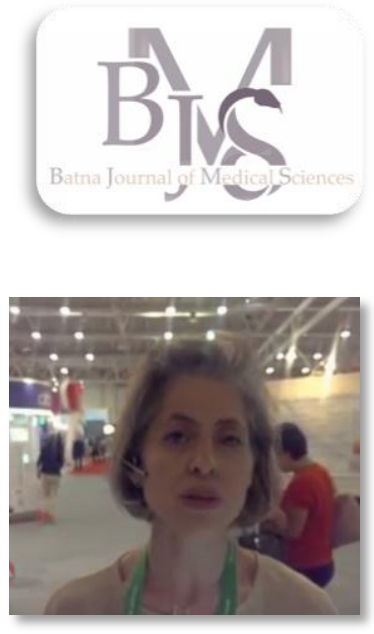

Correspondance à :

Amina ABDESSEMED

aminaabdessemed@yahoo.fr

\section{Brèves de I'EULAR 2015}

\section{Sclérodermie systémique}

Amina Abdessemed (Alger- Algérie)

\title{
Points forts
}

- Les patients traités par sildénafil font moins d’ulcères digitaux ischémiques ;

- Le macitentan, antagoniste des récepteurs de l'endothéline, n'est pas recommandé dans le traitement préventif des ulcères digitaux de la sclérodermie systémique ;

- Le riociguat pourrait améliorer le phénomène de Raynaud primitif et secondaire à la sclérodermie systémique ;

- Amélioration du score de Rodnan modifié et stabilisation de la capacité vitale forcée chez les patients atteints d'une sclérodermie systémique cutanée diffuse récente et traités par tocilizumab ;

- Nouvelles recommandations EULAR pour le traitement de la sclérodermie systémique.

Efficacité du sildénafil dans le traitement des ulcères digitaux de la sclérodermie systémique: Sildenafil Effect on Digital Ulcer Healing in sClerodErma : étude SEDUCE

La sclérodermie systémique est une maladie complexe dont la vasculopathie est un des éléments. Le syndrome de Raynaud y est quasiconstant (Figures 1). Durant le cours évolutif de la maladie, un malade sur deux a développé, développe ou développera au moins un ulcère digital (UD) (Figures 2). Les UD sont responsables de handicap fonctionnel et d'une mauvaise qualité de vie. Le sildénafil, inhibiteur de la phosphodiestérase-5 (PDE-5) augmentele taux intracellulaire de guanosine monophosphate dans la cellule musculaire lisse des vaisseaux induisant une vasodilatation. Ce produit, utilisé dans le traitement de la dysfonctionérectile a été testé dans l'hypertension artérielle pulmonaire primitive et secondaire de la ScS.

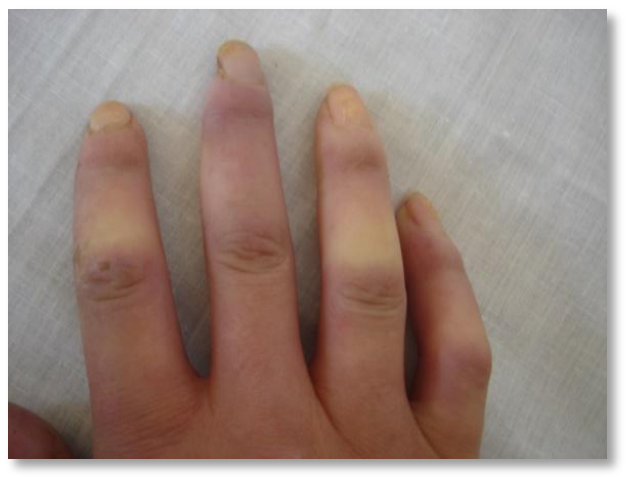

Figure 1 : phénomène de Raynaud

Abdessemed A. Brèves de l'EULAR 2015. Sclérodermie systémique. Batna J Med Sci 2015;2:S18-S20.

Hachulla et coll [0P0058] ont évalué l'efficacité du sildénafil dans la cicatrisation des UD ischémiques de la ScS dans un essai contrôlé, patients avaient reçu du sildénafil à raison de $20 \mathrm{mg}$ trois fois par jour et 41 du placebo. Le principal critère de jugement était le délai de cicatrisation de chaque ulcère. Les patients ont été évalués aux semaines 8 et 12 . Il y avait 89 ulcères digitaux dans le groupe sildénafil et 103 dans le groupe placebo. Sur l'objectif principal, il a été retrouvé un bénéfice en faveur du sildénafil avec un Hazard-Ratio à 1,33 dans un modèle non ajusté pour le nombre d'ulcères digitaux et 1,27 dans un modèle ajusté pour le nombre d'ulcère digitaux (IC $95 \%=0,88$ 2,00 et 0,85 - 1,89 respectivement) mais la différence n'était pas significative. Le nombre d'ulcères digitaux par patient était significativement inférieur dans le groupe sildénafil comparativement au groupe placebo à la semaine $8(1,23 \pm 1,61$ versus $1,79 \pm 2,40$; $\mathrm{p}=0,04)$ et $12(0,8 \pm 1,62$ versus $1,51 \pm 2,68$; $\mathrm{p}$ $=0,01$ ).

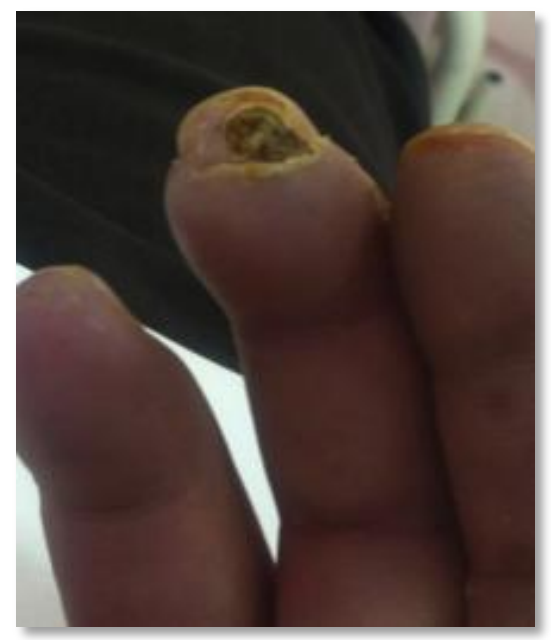

Figure 2 : ulcère digital ischémique randomisé, double aveugle versus placebo. 42
La figure 3 montre les taux de cicatrisation aux semaines 4, 8 et 12. Si l'objectif principal n'a pas été atteint c'est que le taux de cicatrisation à la semaine 12 était largement au-delà de ce 
qui était attendu dans le groupe placebo (66\%, alors que l'hypothèse initiale était de $30 \%$ ). Dans le sous-groupe de patients qui étaient sous bosentan au moment de l'inclusion (un tiers des patients), l'objectif principal était atteint en intention de traiter en faveur de la combinaison sildénafil + bosentan. Le sildénafil est recommandé dans le traitement des UD de la ScS en association aux soins locaux. Avant d'être recommandée, la combinaison inhibiteurs de la phosphodiestérase et antagonistes des récepteurs de l'endothéline doit être évaluée dans un essai thérapeutique prospectif.

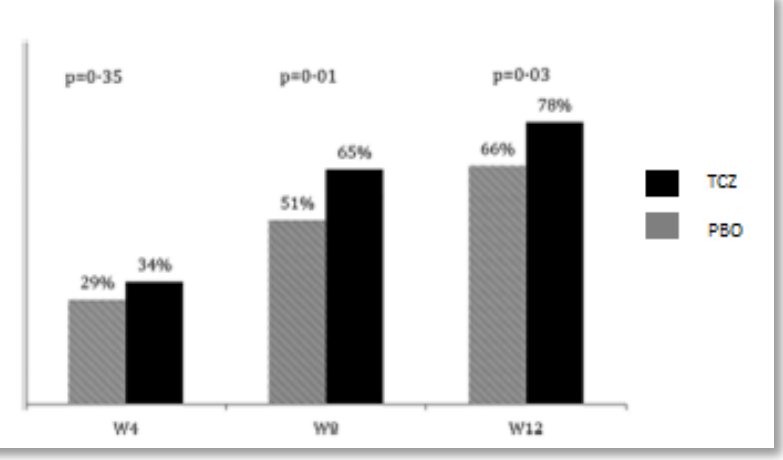

Figure 3: taux de cicatrisation aux semaines 4,8 et 12 dans les groupes tocilizumab et placebo

Le macitentan dans le traitement des ulcères digitaux de la sclérodermie systémique : résultats de deux essais contrôlés, randomisés, DUAL 1 et DUAL 2

Les ulcères digitaux (UD) de la $\mathrm{ScS}$ sont le plus souvent multiples et récidivants. Ils peuvent se compliquer d'infection, d'ostéite, de nécrose digitale et d'amputation. Leur cicatrisation est lente. L'endothéline 1 est un petit peptide synthétisé par de nombreux types cellulaires dont les cellules endothéliales, capables d'interagir avec des récepteurs de type $\mathrm{A}$ ou $\mathrm{B}$. Les effets produits dépendent du récepteur mais aussi du tissu cible et de la concentration locale en endothéline 1. Le taux d'endothéline est élevé chez les patients atteints de sclérodermie systémique et particulièrement chez les patients ayant des ulcères digitaux. L'endothéline 1a des propriétés vasoconstrictrices et pourrait également stimuler la prolifération de fibroblastes et la synthèse de collagène. Le bosentan est un inhibiteur des récepteurs de type $A$ et $B$ de l'endothéline 1 (ERA). Deux études contrôlées, randomisées, en double aveugle, versus placebo, (RAPIDS 1 et RAPIDS 2), ont montré une diminution significative du nombre de nouveaux ulcères digitaux chez les patients traités par bosentan. Ce dernier a obtenu l'AMM dans la prévention de la récidive d'ulcères digitaux de la ScS par contre il n'a aucun effet sur la cicatrisation. Le macitentan est un autre antagoniste des récepteurs de l'endothéline qui a obtenu l'AMM dans le traitement de l'HTAP de la ScS. D Khanna et coll [OP0056] rapportent les résultats de deux études contrôlées, randomisées, double aveugle versus placebo : DUAL 1 (289 patients) et DUAL 2 (265patients). L'objectif de l'étude était d'évaluer l'efficacité, l'innocuité et la tolérance du macitentan dans le traitement des UD de la ScS. Le Critère de jugement principal était la réduction du nombre de nouveaux UD. Les patients ont été randomisés en 3 bras : macitentan $3 \mathrm{mg} / \mathrm{j}$, macitentan $10 \mathrm{mg} / \mathrm{j}$, et placebo. L'évaluation s'est faite à la semaine 16. Le nombre de nouveaux ulcères digitaux était identique dans les trois groupes (Tableau 1). L'objectif principal n'a pas été atteint. Le macitentan était bien toléré, les principaux effets secondaires étaient: céphalées, œdèmes périphériques, anémie, pharyngite, infection respiratoire, diarrhée et ulcérations cutanées. Les auteurs concluent que le macitentan ne peut être recommandé dans la prévention de nouveaux ulcères digitaux de la ScS.

Tableau 1 : résultats des études DUAL 1 et DUAL 2

\begin{tabular}{|c|c|c|c|c|}
\hline & \multicolumn{2}{|c|}{ DUAL-1 } & \multicolumn{2}{|c|}{ DUAL-2 } \\
\hline & $\begin{array}{l}\text { Macitentan 3mg } \\
\text { vs placebo } \\
\text { TE (95\% CI) }\end{array}$ & $\begin{array}{l}\text { Macitentan } 10 \mathrm{mg} \\
\text { vs placebo } \\
\text { TE }(95 \% \mathrm{Cl})\end{array}$ & $\begin{array}{l}\text { Macitentan 3mg } \\
\text { vs placebo } \\
\text { TE (95\% CI) }\end{array}$ & $\begin{array}{c}\text { Macitentan } 10 \mathrm{mg} \\
\text { vs placebo } \\
\text { TE }(95 \% \mathrm{Cl})\end{array}$ \\
\hline $\begin{array}{l}\text { Cumulative number of } \\
\text { new DUs at Week } 16\end{array}$ & $\begin{array}{c}1.10(0.66,1.83) \\
p=0.706\end{array}$ & $\begin{array}{c}1.27(0.76,2.11) \\
p=0.360\end{array}$ & $\begin{array}{c}1.19(0.77,1.86) \\
p=0.434\end{array}$ & $\begin{array}{c}1.21(0.77-1.89) \\
p=0.407\end{array}$ \\
\hline $\begin{array}{l}\leqslant 3 \text { OUs at } \\
\text { randomisation }\end{array}$ & $\begin{array}{c}1.09(0.58,2.05) \\
p=0.798\end{array}$ & $\begin{array}{c}1.40(0.75,2.60) \\
p=0.291\end{array}$ & $\begin{array}{c}1.02(0.54,1.95) \\
p=0.943\end{array}$ & $\begin{array}{c}1.14(0.60,2.17) \\
p=0.685\end{array}$ \\
\hline $\begin{array}{l}>3 \text { DUs at } \\
\text { randomisation }\end{array}$ & $\begin{array}{c}1.32(0.72,2.43) \\
p=0.770\end{array}$ & $\begin{array}{c}1.20(0.64,2.25) \\
p=0.946\end{array}$ & $\begin{array}{c}1.48(0.83,2.67) \\
p=0.187\end{array}$ & $\begin{array}{c}1.32(0.73,2.40) \\
p=0.365\end{array}$ \\
\hline
\end{tabular}

calculated using a negative binomial model and adjusted for the number of DUs at baseline.

Traitement du phénomène de Raynaud par le riociguat, un activateur de la guanylate cyclase soluble

Le syndrome de Raynaud est un acrosyndrome vasculaire, il peut être primitif (idiopathique) ou secondaire à une connectivite. Huntgeburth et coll [FRI0444] ont évalué l'efficacité et la tolérance du riociguat, molécule activatrice de la guanylate cyclase soluble, dans le traitement du syndrome de Raynaud. Ont été inclus les patients âgés de 18 à 70 ans, ayant un syndrome de Raynaud primitif ou secondaire à une ScS limitée ou diffuse ou à un syndrome de chevauchement et évoluant depuis une année et plus. Les patients ont été randomisés pour recevoir une dose unique de $2 \mathrm{mg}$ de riociguat ou du placebo. Le principal critère de jugement était le flux sanguin mesuré au niveau de l'index droit par laser après exposition au froid à l'inclusion et deux heures après administration de la molécule. Un patient était considéré comme répondeur s'il avait une amélioration du flux $\geq 10 \%$ vs baseline deux heures après la prise de riociguat. 20 patients ont été inclus. 85\% étaient des femmes, l'âge moyen était de 52 ans. La majorité d'entre eux avaient un syndrome de Raynaud secondaire, 10 patients avaient une ScS cutanée diffuse. 12/20 (60\%) étaient répondeurs, les meilleures réponses étaient observées dans les groupes syndrome de Raynaud primitif et ScS cutanée limitée (Tableau 2). 5 effets secondaires ont été rapportés dans le groupe riociguat (céphalées $n=4$ et dyspepsie $n=1$ ) et 1 dans le groupe placebo.

Tableau 2: réponse au riociguat chez les patients ayant un phénomène de Raynaud

\begin{tabular}{llllll} 
& $\begin{array}{l}\text { Primary } \\
\text { (idiopathic) }\end{array}$ & $\begin{array}{l}\text { Diffuse } \\
\text { cutaneous } \\
\text { SSc }\end{array}$ & $\begin{array}{l}\text { Limited } \\
\text { cutaneous } \\
\text { SSc }\end{array}$ & $\begin{array}{l}\text { SSc } \\
\text { overlap } \\
\text { syndrome }\end{array}$ & Total \\
\hline $\begin{array}{l}\text { No. of } \\
\text { patients }\end{array}$ & 3 & 10 & 5 & 2 & 20 \\
$\begin{array}{l}\text { Responders, } \\
\mathrm{n}(\%)^{\mathrm{a}}\end{array}$ & $2(67)$ & $5(50)$ & $4(80)$ & $1(50)$ & $12(60)$ \\
\hline
\end{tabular}

a Placebo-corrected blood flow after cold exposure increased by $\geq 10 \%$ vs baseline at 2 hours after drug intake. SSc, scleroderma. 
Efficacité et tolérance du tocilizumab sous-cutané chez des patients adultes atteints de sclérodermie systémique : résultats de l'étude FASSCINATE

La sclérodermie systémique (ScS) est une affection généralisée du tissu conjonctif caractérisée par des atteintes polyviscérales. Cette maladie se distingue des autres connectivites en raison d'un processus pathologique original : elle est marquée par des anomalies microvasculaires, initialement spastiques et en partie réversibles, une inflammation avec participation dysimmunitaire, puis une activation fibroblastique qui conduit à la fibrose. Le tocilizumab est un antagoniste du récepteur de l'interleukine6 (IL-6). Plusieurs études rapportent le rôle de l'IL-6 dans la pathogénie de la sclérodermie systémique. Dans les modèles murins de ScS l'inhibition du récepteur de l'IL-6 (IL-6R) empêche le développement de la fibrose cutanée et permet la régression de la fibrose déjà installée.D. Khanna et coll [0P0054] ont évalué l'efficacité et la tolérance du tocilizumab chez des patients atteints de ScS dans une étude contrôlée, randomisée, en double aveugle versus placebo (Etude phase II). Ont été inclus les patients âgés de 18 ans et plus, atteints d'une ScS répondant aux critères ACR de 1980, évoluant depuis moins de 5 ans et ayant un score de Rodnan modifié (mRSS) $\geq 15$ et un syndrome inflammatoire biologique. 87 patients ont été randomisés pour recevoir soit du tocilizumab (TCZ) $162 \mathrm{mg}$ (43 patients) soit un placebo (PBO) (44 patients) en sous-cutané une fois par semaine pendant 48 semaines. Le critère de jugement principal était la variation du mRSS moyen à la semaine 24 . Ont également été évalués le mRSS moyen à la semaine 48 , la capacité vitale forcée (CVF), le HAQ-DI et le score FACIT-fatigue à la semaine 24 et 48 . L'amélioration du score de Rodnan modifié ( -3.9 groupe TCZ vs -1.2 groupe PBO) et des scores HAQ-DI et FACIT était plus importante dans le groupe TCZ comparé au groupe PBO mais la différence n'était pas statistiquement significative. Douze pour cent des patients sous tocilizumab avaient une amélioration du score de Rodnan d'au moins $60 \%$ vs $0 \%$ dans le groupe placebo $(p=0,03)$.La dégradation de la fonction respiratoire, évaluée par la mesure de la CVF était plus fréquente dans le groupe PBO. Une diminution de la CVF de plus de $10 \%$ était survenue chez $10 \%$ des patients TCZ contre $23 \%$ des patients $\mathrm{PBO}$ à la semaine 48 . Les résultats de l'étude sont résumés dans le tableau 3 . Le nombre d'effets secondaires était similaire dans les deux groupes. Un décès est survenu dans le groupe PBO et 3 dans le groupe TCZ à la semaine 48. Un décès était dû à une infection pulmonaire sévère dans le groupe TCZ.

Tableau 3 : principaux résultats de l'étude FASSCINATE

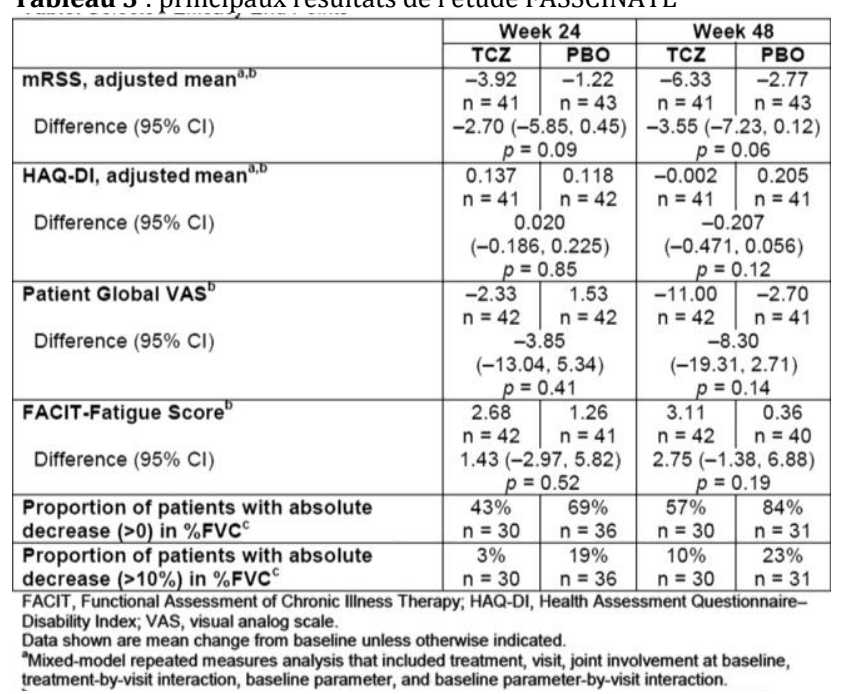

Mise à jour des recommandations EULAR pour le traitement de la sclérodermie systémique [0P0061]

Les dernières recommandations pour le traitement de la ScS remontent à 2009, l'objectif de ce travail était de les mettre à jour au vu des derniers essais thérapeutiques rapportés. Le groupe de travail comportait 30 experts, 1 épidémiologiste et des représentants des associations de patients. Les centres EUSTAR ont également été associés.Une revue exhaustive de la littérature était réalisée pour chaque question et des recommandations émises et gradées de $\mathrm{A}$ à $\mathrm{D}$ en fonction de leur niveau de preuve (A: essai randomisé et $\mathrm{D}$ : avis d'expert). 16 recommandations ont été retenues (14 en 2009). Les principales différences ont été rapportées lors du congrès EULAR en attendant la publication des recommandations. Les inhibiteurs de la phosphodiéstérase-5 et la fluoxétine (inhibiteur sélectif de la recapture de la sérotonine) peuvent être utilisés, après échec des inhibiteurs calciques, dans le traitement du phénomène de Raynaud. Pour les ulcères digitaux ischémiques, peuvent être prescrits en deuxième intention (après l'iloprost) les inhibiteurs de la phosphodiéstérase-5. Le Bosentan est recommandé dans le traitement préventif des UD récidivants après échec des autres thérapeutiques. Concernant l'hypertension artérielle pulmonaire, sont recommandés les antagonistes des récepteurs de l'endothéline : ambrisentan, macitentan, bosentan; les inhibiteurs de la phosphodiéstérase-5 : sildenafil, tadalafil ; le riociguat, une molécule activatrice de la guanylate cyclase soluble et les analogues de la prostacycline : epoprostenol, iloprost, treprostinil. La Greffe de cellules souches hématopoiétiques est recommandée dans le traitement de certaines formes cutanées diffuses, sévères rapidement progressives. 